\title{
Niereninsuffizienz bei Patienten mit einem thromboembolischen Ereignis: Prävalenz und klinische Implikationen.
}

\author{
Eine systematische Übersicht der Literatur
}

T. Wilke ${ }^{1}$, M. Wehling ${ }^{2}$, S. Amann ${ }^{3}$, R. M. Bauersachs ${ }^{4}$, B. Böttger ${ }^{1}$

Institute

1 Institut für Pharmakoökonomie und Arzneimittellogistik an der Hochschule Wismar

2 Klinische Pharmakologie/Zentrum für Gerontopharmakologie, Medizinische Fakultät Mannheim, Universität Heidelberg,

3 Staedtisches Klinikum Muenchen $\mathrm{GmbH}$, Krankenhausapotheke Schwabing

4 Medizin IV-Vaskuläre Medizin, Max Ratschow Klinik, Klinikum Darmstadt $\mathrm{GmbH}$

\section{Korrespondenz}

Prof. Dr. rer. oec.

Thomas Wilke

Institut für Pharmakoökonomie und Arzneimittellogistik an der Hochschule Wismar Philipp-Müller-Str. 12 23966 Wismar Tel. +4938417581014 Fax +4938417581011 thomas.wilke@ipam-wismar. de

\section{Zusammenfassung}

Hintergrund und Fragestellung | Die Bestimmung der Nierenfunktion ist von besonderer Bedeutung bei Auswahl und Dosierung der initialen Antikoagulation von Patienten mit einem thromboembolischen Ereignis, da zahlreiche Antikoagulanzien über die Niere ausgeschieden werden. Insbesondere für Patienten, die eine schwer eingeschränkte Nierenfunktion aufweisen, steigt das Risiko einer Akkumulation der Wirkstoffe und somit das Risiko von Blutungskomplikationen. In den aktuellen Fachinformationen der zur Therapie zugelassenen Wirkstoffe sowie den aktuellen AWMF-Leitlinien wird diesem Aspekt besondere Aufmerksamkeit gewidmet. So sind einige Wirkstoffe in dieser Patientengruppe kontraindiziert, für einige Wirkstoffe wird eine erhöhte Kontrollintensität (anti-Xa) empfohlen und für den Wirkstoff Enoxaparin ist eine Dosisreduktion vorgesehen. Ziel dieser Übersichtsarbeit ist die Beantwortung folgender Fragen:

- Wie hoch ist die Prävalenz der Niereninsuffizienz bei Patienten mit venöser Thromboembolie (VTE)?

- Welche Daten zur initialen Antikoagulationsbehandlung von niereninsuffizienten VTE-Patienten und zu mit der Behandlung korrespondierenden klinischen Ergebnissen (Rezidive, Blutungsereignisse und Mortalität) sind verfügbar?

Methodik I Es wurde eine systematische Datenbanksuche und eine Bewertung der verfügbaren englisch- und deutschsprachigen Literatur des Zeitraumes von Januar 2004 bis Januar 2014 durchgeführt. Dabei wurden lediglich Publikationen in die Übersichtsarbeit aufgenommen, die als quantitative Forschung bezeichnet werden können.

Ergebnisse I Insgesamt konnten 1135 Studien identifiziert werden, von denen letztlich 37 in die Übersichtsarbeit eingeschlossen wurden.

\section{Einführung}

Die Bestimmung der Nierenfunktion (NF) stellt einen wichtigen Bestandteil in der Anamnese eines Patienten dar. Von besonderer Bedeutung ist die Beurteilung der NF bei Auswahl und Dosierung einer Pharmakotherapie, da zahlreiche Arznei-
Die Prävalenz einer Kreatinin-Clearance $(\mathrm{KrCl})$ unter $60 \mathrm{ml} / \mathrm{min}$ lag zwischen 12,3 und 71,9\% der VTE-Patienten. Die Prävalenz einer $\mathrm{KrCl}$ unter $30 \mathrm{ml} / \mathrm{min}$ betrug 3,3-13,6\%. Die großen Unterschiede sind ganz wesentlich durch verschiedene Patientenkollektive zu erklären.

Eine $\mathrm{KrCl}<30 \mathrm{ml} /$ min war ein unabhängiger Prädiktor für Mortalität und fatale Lungenembolie, evtl. auch für schwere Blutungen bei der Therapie von VTE-Patienten. Dabei ist sie vermutlich auch schon ein Prädiktor für das Auftreten eines VTEEreignisses.

Die Daten zeigen auch, dass zahlreiche Antikoagulanzien zur Akkumulation neigen und so, beispielhaft, eine Standarddosis Enoxaparin zu höheren Blutungsraten in der VTE-Therapie führt als eine angepasste Dosis Enoxaparin. Zu anderen niedermolekularen Heparinen (NMH) und zu unfraktionierten Heparinen (UFH) gab es weitaus weniger umfangreiche Daten. Allein zur Sicherheit von Certoparin und Tinzaparin konnten eigene Studien in der betrachteten speziellen Patientengruppe identifiziert werden.

Keine der identifizierten Studien zeigt Effektivitäts- und/oder Sicherheitsvorteile der UFH- im Vergleich zur NMH-Therapie bei VTE-Patienten mit stark eingeschränkter Nierenfunktion; vielmehr kann nach aktueller Studienlage deren Nachteiligkeit angenommen werden. Die Evidenz beruht allerdings auf observationalen Studien bzw. Registerdaten.

Schlussfolgerung I Die Kenntnis der Fachinformation der initialen Antikoagulanzien ist unerlässlich, da diese nähere Auskünfte zu Dosierungsschemata bzw. zur Kontraindikation einiger Substanzen präzisieren. Die aktuelle AWMF-Leitlinie ist hinsichtlich der Empfehlung von UFHs als Option erster Wahl bei $\mathrm{KrCl}<30 \mathrm{ml} / \mathrm{min}$ zu diskutieren.

stoffe über die Niere ausgeschieden werden [1] Dies gilt auch im Fall der initialen Antikoagulation von Patienten nach einer venösen Thromboembolie (VTE) [2]. In Deutschland werden jährlich 64000-92000 VTE-Patienten initial in Akutkrankenhäusern behandelt [3]. 


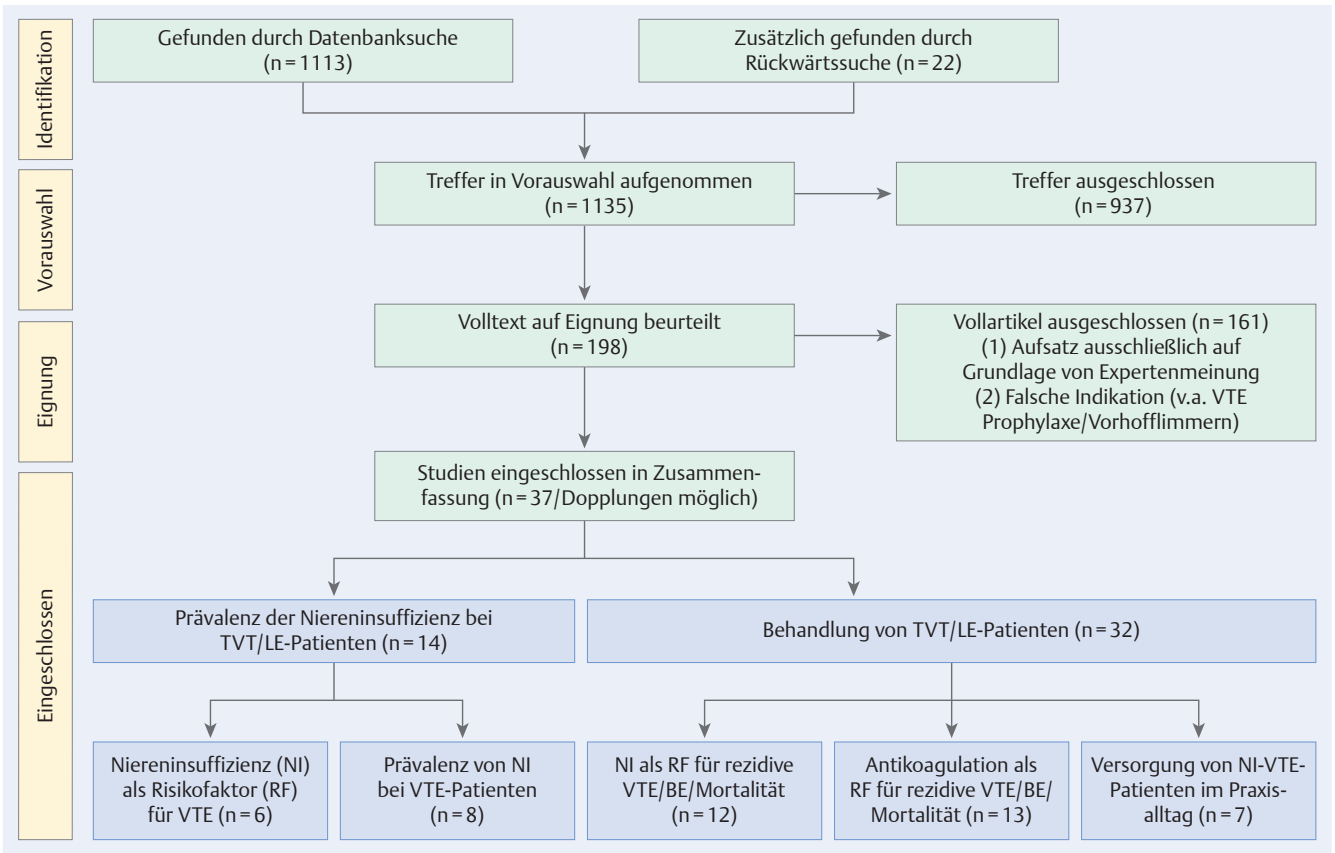

Behandlungsleitlinien empfehlen im Fall einer akut auftretenden VTE die sofortige therapeutische Antikoagulation, vorzugsweise mit niedermolekularen Heparinen (NMH) oder Fondaparinux [2]. Sollten VTE-Patienten allerdings unter einer mäßig eingeschränkten NF (Kreatinin-Clearance [ $\mathrm{KrCl}]$ $30-60 \mathrm{ml} / \mathrm{min}$ ) oder stark eingeschränkten NF bzw. Niereninsuffizienz $(\mathrm{KrCl}<30 \mathrm{ml} / \mathrm{min})$ leiden, steigt das Risiko der Akkumulation der angewendeten therapeutischen Antikoagulanzien und damit das Blutungsrisiko der Patienten [4-15]. Folgerichtig empfehlen sowohl Leitlinien als auch Produktinformationen der Antikoagulanzien spezifische Behandlungsmaßnahmen, insbesondere die Dosisanpassung, für diese Patientengruppe [2].

Trotz der bekannten Bedeutung der NF bei der initialen Antikoagulation von VTE-Patienten sind Zahlen zur Prävalenz einer Niereninsuffizienz bei dieser Patientengruppe sowie Daten zur Behandlung der Patienten kaum bekannt. Zudem werden Patienten mit einer $\mathrm{KrCl}<30 \mathrm{ml} / \mathrm{min}$ in aller Regel aus klinischen Studien ausgeschlossen [16, 17]. Ziel dieser Übersichtsarbeit war es deshalb, verfügbare publizierte Daten zu zwei Fragestellungen zusammenzufassen:

- Wie hoch ist die Prävalenz einer Niereninsuffizienz bei VTE-Patienten?

- Welche Daten zur initialen Antikoagulationsbehandlung von niereninsuffizienten VTE-Patienten und zu mit der Behandlung korrespondierenden klinischen Ergebnissen (Rezidive, Blutungsereignisse und Mortalität) sind verfügbar?

\section{Methodik}

Es wurde eine systematische Datenbanksuche (PubMed/Medline) der verfügbaren englisch- und deutschsprachigen Literatur des Zeitraumes von Januar 2004 bis Januar 2014 in Bezug auf relevante Suchbegriffe durchgeführt (siehe OnlineTabelle sTab. 1). Dabei wurden lediglich Publikationen in die Übersicht aufgenommen, die als quantitative Forschung bezeichnet werden können; qualitative Expertenmeinungen wurden ausgeschlossen.

Die mittels Datenbanksuche identifizierten Beiträge wurden anhand der Abstracts und anschließend anhand des Volltextes auf ihre Relevanz im Kontext dieser Übersicht geprüft. Innerhalb des Screenings wurden Duplikate sowie Aufsätze, die aufgrund der Einschlusskriterien als ungeeignet erachtet wurden, ausgeschlossen. Sämtliche Publikationen wurden von mindestens zwei Autoren detailliert ausgewertet; fachliche Differenzen wurden im Konsens geklärt.

Die Analyse erfolgte in Übereinstimmung mit dem standardisierten Vorgehen laut PRISMAStatement [18]. Eine eigene Meta-Analyse wurde aufgrund der Heterogenität der einzelnen Studien, der studienspezifisch unterschiedlichen Endpunkte und der teilweise qualitativen Zielsetzung dieser Übersichtsarbeit nicht durchgeführt.

\section{Ergebnisse}

Insgesamt konnten durch die Suche 1135 Beiträge identifiziert werden (siehe Online-Tabelle - sTab. 1). Nach dem Bereinigen um Duplikate, Sichtung und Studium der Treffer wurden letztlich 37 Studien in die Übersichtsarbeit eingeschlossen ( $\triangleright$ Abb. 1, $\triangleright$ Tab. 1). Die relevanten Studien wurden hinsichtlich der Ergebnisse aus den Themenbereichen „Prävalenz der Niereninsuffizienz bei
Abb. 1 PRISMA Flowchart zum durchgeführten Review. 
Tab. 1 Prävalenz von Niereninsuffizienz bei TVT / LE-Patienten

VTE-Patienten“ und „Behandlung von VTE-Patienten" analysiert.

Prävalenz von Niereninsuffizienz bei VTE-Patienten I Die Literatur zur Prävalenz einer Niereninsuffizienz bei VTE-Patienten beschäftigt sich mit zwei generellen Themenbereichen:

- der Darstellung einer Niereninsuffizienz als Risikofaktor für das Auftreten einer VTE sowie

- der Prävalenz einer Niereninsuffizienz bei diagnostizierten VTE-Patienten.

Obwohl der zweite Themenbereich im Fokus dieser Übersicht steht, sollen kurz auch die publizierten Daten zum ersten Themenbereich dargestellt werden.

Die in diesem Teil inkludierten 6 Publikationen zeigen überwiegend, dass Niereninsuffizienz ein unabhängiger Risikofaktor für das Auftreten einer VTE ist. In einer Übersicht zeigten Mahmoodi et al., dass eine $\mathrm{KrCl}<30 \mathrm{ml} / \mathrm{min}$, verglichen mit einer $\mathrm{KrCl}>100 \mathrm{ml} / \mathrm{min}$, mit einem Hazard Ratio (HR) von 1,95 für das Auftreten einer VTE verbunden ist [19]. Folsom et al. kamen in einer prospektiven Kohortenstudie zum Ergebnis eines 1,6-fachen VTE-Risikos bei einer $\mathrm{KrCl}$ von 15-59 ml/ $\mathrm{min}$ [20]. Diese Ergebnisse werden bestätigt durch Kumar et al. in einer Analyse eines Registers [21] und in einer von Wattanakit et al. durchgeführten Übersichtsarbeit [22]. Cook et al. zeigten, dass terminale Niereninsuffizienz ein Risikofaktor für das Auftreten einer TVT während einer stationären Behandlung ist (HR 3,7) [23]. Königsbrügge et al. analysierten speziell die Gruppe onkologischer Patienten und fanden in diesem Kollektiv keinen gesicherten
Zusammenhang zwischen NF und VTE-Inzidenz. Aufgrund des sehr geringen Anteils von Patienten mit einer $\mathrm{KrCl}<30 \mathrm{ml} / \mathrm{min}$ gilt diese Aussage allerdings nur oberhalb dieser Grenze [24].

Die in die Übersicht eingeschlossenen 8 Publikationen zur Prävalenz der Niereninsuffizienz bei VTE-Patienten verwenden unterschiedliche Definitionen einer Niereninsuffizienz: Am gebräuchlichsten sind $\mathrm{KrCl}-\mathrm{Grenzwerte}$ von $60 \mathrm{ml} / \mathrm{min}$ oder $30 \mathrm{ml} / \mathrm{min}$.

In einer allgemeinen Analyse von Patienten über 65 Jahren berichten Kurtal et al. einen Anteil von 43-72\%derPatienten, dievoneiner $\mathrm{KrCl}<60 \mathrm{ml} / \mathrm{min}$ betroffen sind. Die unterschiedlichen Prozentsätze sind auf unterschiedliche NF-Schätzformeln zurückzuführen [25]. Die Studienkohorte von Parikh et al. wies einen etwas geringeren Anteil von Patienten mit einer $\mathrm{KrCl}<60 \mathrm{ml} / \mathrm{min}$ in Höhe von 36,7\% auf. Allerdings wurden Dialysepatienten in dieser Studie exkludiert [26]. Trujillo-Santos und Kollegen berichteten, dass $18,1 \%$ der VTE-Patienten in ihrer Studie eine $\mathrm{KrCl}<60 \mathrm{ml} / \mathrm{min}$ aufwiesen [27]. Cook et al. wiesen in einer retrospektiven Studie von 524 VTE-Patienten einen Anteil von 25\% mit einer $\mathrm{KrCl}<60 \mathrm{ml} /$ min sowie einen Anteil der Patienten mit einer $\mathrm{KrCl}<30 \mathrm{ml} /$ min von 5\%aus [23]. Monreal et al. kamen in einer Analyse der Daten des VTERIETE-Registers zum Ergebnis, dass 12,3\% der Patienten eine $\mathrm{KrCl}<60 \mathrm{ml} / \mathrm{min}$ aufweisen [11]. Ebenfalls auf Basis der Daten des RIETE-Registers berichteten Falgá et al. für 1037 der 18251 eingeschlossenen VTE-Patienten eine $\mathrm{KrCl}<30 \mathrm{ml} / \mathrm{min}$ $(5,7 \%)$ [7]. In einer Stichprobe von 798 intensiv-

\begin{tabular}{|c|c|c|c|c|c|c|c|c|}
\hline \multirow[t]{2}{*}{ Autoren } & \multicolumn{3}{|c|}{ Patienten } & \multirow{2}{*}{$\begin{array}{l}\text { Behandlungs- } \\
\text { sektor }\end{array}$} & \multicolumn{4}{|c|}{ Anteil der Patienten mit jeweiliger $\mathrm{KrCl}$} \\
\hline & $\mathbf{n}$ & Alter & $\begin{array}{l}\text { Anteil } \\
\text { Frauen }\end{array}$ & & $<15 \mathrm{ml} / \mathrm{min}$ & $15-29 \mathrm{ml} / \mathrm{min}$ & $30-59 \mathrm{ml} / \mathrm{min}$ & $60-89 \mathrm{ml} / \mathrm{min}$ \\
\hline $\begin{array}{l}\text { Al-Dorzi } \\
\text { et al. [28] }\end{array}$ & $\begin{array}{l}798 \\
\text { TVT/LE }\end{array}$ & $\begin{array}{l}\varnothing 50,2 \\
\text { Jahre }\end{array}$ & $33,0 \%$ & $\begin{array}{l}\text { Stationär } \\
\text { (Intensivstation) }\end{array}$ & $10,1 \%$ & - & - & - \\
\hline $\begin{array}{l}\text { Boettger } \\
\text { et al. [29] }\end{array}$ & $\begin{array}{l}4554 \\
\text { TVT/LE }\end{array}$ & $\begin{array}{l}\emptyset 67,8 \\
\text { Jahre }\end{array}$ & $53,0 \%$ & Stationär & \multicolumn{2}{|c|}{$7,4 \%$} & - & - \\
\hline $\begin{array}{l}\text { Cook } \\
\text { et al. [23] }\end{array}$ & $\begin{array}{l}524 \\
\text { TVT/LE }\end{array}$ & $\begin{array}{l}\emptyset 62,4 \\
\text { Jahre }\end{array}$ & $53,0 \%$ & Stationär & \multicolumn{2}{|c|}{$5,0 \%$} & $20,0 \%$ & - \\
\hline $\begin{array}{l}\text { Falgá } \\
\text { et al. [7] }\end{array}$ & $\begin{array}{l}18251 \\
\text { TVT/LE }\end{array}$ & $\begin{array}{l}52,7 \% \\
>70 \text { Jahre }\end{array}$ & $50,4 \%$ & $\begin{array}{l}\text { Stationär + } \\
\text { Ambulant }\end{array}$ & \multicolumn{2}{|c|}{$5,7 \%$} & & \\
\hline \multirow{2}{*}{$\begin{array}{l}\text { Kurtal } \\
\text { etal. [25] }\end{array}$} & \multirow[t]{2}{*}{221} & \multirow{2}{*}{$\begin{array}{l}\varnothing 80,3 \\
\text { Jahre }\end{array}$} & \multirow[t]{2}{*}{-} & \multirow[t]{2}{*}{ Stationär } & \multicolumn{2}{|c|}{$3,3 \%-13,6 \%$} & - & - \\
\hline & & & & & \multicolumn{3}{|c|}{$43,0 \%-71,9 \%$} & - \\
\hline $\begin{array}{l}\text { Monreal } \\
\text { et al. [11] }\end{array}$ & $\begin{array}{l}10526 \\
\text { VTE }\end{array}$ & $\begin{array}{l}65,0 \% \\
>65 \text { Jahre }\end{array}$ & $50,1 \%$ & $\begin{array}{l}\text { Stationär + } \\
\text { Ambulant }\end{array}$ & \multicolumn{2}{|c|}{$5,6 \%$} & $6,7 \%$ & - \\
\hline $\begin{array}{l}\text { Parikh } \\
\text { et al. [12] }\end{array}$ & $\begin{array}{l}1509 \\
\text { VTE }\end{array}$ & $\begin{array}{l}\varnothing 65,4 \\
\text { Jahre }\end{array}$ & $54,9 \%$ & $\begin{array}{l}\text { Stationär + } \\
\text { Ambulant }\end{array}$ & \multicolumn{2}{|c|}{$8,1 \%$} & $28,6 \%$ & $38,1 \%$ \\
\hline $\begin{array}{l}\text { Trujillo- } \\
\text { Santos } \\
\text { etal. [27] }\end{array}$ & $\begin{array}{l}38531 \\
\text { TVT/LE }\end{array}$ & $\begin{array}{l}\varnothing 60,9 \\
\text { Jahre }\end{array}$ & $50,9 \%$ & $\begin{array}{l}\text { Stationär + } \\
\text { Ambulant }\end{array}$ & \multicolumn{2}{|c|}{$5,8 \%$} & $12,3 \%$ & - \\
\hline
\end{tabular}




\begin{tabular}{|c|c|c|c|c|c|c|}
\hline \multirow{2}{*}{ Wirkstoff } & \multirow{2}{*}{ Indikation } & \multicolumn{5}{|c|}{ Kreatinin-Clearance-Bereich (in $\mathrm{ml} / \mathrm{min}$ ) } \\
\hline & & 20 & 40 & 80 & \multicolumn{2}{|c|}{90} \\
\hline & & \multicolumn{5}{|c|}{ 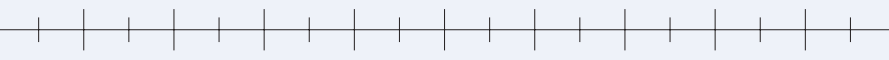 } \\
\hline Tinzaparin & TVT, LE & $\begin{array}{l}\text { Kontrolle aXa-Spiegel + } \\
\text { erhöhte Vorsicht }\end{array}$ & \multicolumn{4}{|c|}{ Ohne Einschränkungen } \\
\hline Dalteparin & TVT, LE & $\begin{array}{l}\text { Kontrolle aXa-Spiegel + } \\
\text { erhöhte Vorsicht }\end{array}$ & \multicolumn{4}{|c|}{ Ohne Einschränkungen } \\
\hline \multirow{2}{*}{ Enoxaparin } & \multirow{2}{*}{ TVT, LE } & aXa: $0,4-1,1 \mathrm{IU} / \mathrm{ml}$ & \multirow{2}{*}{\multicolumn{2}{|c|}{ Sorgfältige Überwachung }} & \multirow{2}{*}{\multicolumn{2}{|c|}{$\begin{array}{c}\text { Ohne } \\
\text { Einschränkungen }\end{array}$}} \\
\hline & & Dosisanpassung & & & & \\
\hline Certoparin & TVT & $\begin{array}{l}\text { Therapie laut Fachinforma- } \\
\text { tion nicht empfohlen }\end{array}$ & Anwend & & & $\begin{array}{l}\text { Ohne } \\
\text { Einschr. }\end{array}$ \\
\hline \multirow{2}{*}{ Nadroparin } & \multirow{2}{*}{ TVT } & \multirow{2}{*}{ Kontraindiziert } & \multicolumn{3}{|c|}{ aXa-Spiegel: $0,5-1,2 \mathrm{IU} / \mathrm{ml}$} & Ohne \\
\hline & & & Ggf. Dosisanpa & & & Einschr. \\
\hline \multirow{2}{*}{ Reviparin } & \multirow{2}{*}{ TVT } & \multirow{2}{*}{ Kontraindiziert } & \multicolumn{3}{|c|}{ Anwendung unter erhöhter Vorsicht } & \multirow{2}{*}{$\begin{array}{l}\text { Ohne } \\
\text { Einschr. }\end{array}$} \\
\hline & & & aXa: $0,5-1,2 \mathrm{IU} / \mathrm{ml}$ & & & \\
\hline Fondaparinux & TVT, LE & Kontraindiziert & $\begin{array}{l}\text { Anwendung unter } \\
\text { erhöhter Vorsicht } \\
\text { +Dosisanpassung }\end{array}$ & & igen & \\
\hline UFH & TVT, LE & \multicolumn{4}{|c|}{ Sorgfältige Überwachung der Gerinnungswerte (aPTT) } & $\begin{array}{l}\text { Ohne } \\
\text { Einschr. }\end{array}$ \\
\hline
\end{tabular}

medizinisch behandelten VTE-Patienten wiesen nach Al-Dorzi et al. 10,1\% eine terminale Niereninsuffizienz von $\mathrm{KrCl}<15 \mathrm{ml} / \mathrm{min}$ auf [28]. In einer von uns durchgeführten retrospektiven Patientenaktenstudie von VTE-Patienten in 6 deutschen Akutkrankenhäusern hatten 337 von 4554 Patienten $(7,4 \%)$ eine Nierenfunktion während des Akutaufenthalts von $\mathrm{KrCl}<30 \mathrm{ml} / \min$ [29].

Behandlung niereninsuffizienter TVT/LE-Patienten I Die deutschen Behandlungsleitlinien empfehlen zur initialen therapeutischen Antikoagulation bevorzugt NMH oder Fondaparinux. Es wird explizit auf das Risiko einer Akkumulation der empfohlenen Wirkstoffe bei Patienten mit einer $\mathrm{KrCl}<30 \mathrm{ml} / \mathrm{min}$ eingegangen, indem für diese Patienten die initiale Behandlung mit unfraktionierten Heparinen (UFH) empfohlen wird. Die evtl. vorsichtige bzw. dosisadaptierte Gabe von $\mathrm{NMH}$ hat bei dieser Patientengruppe bislang keinen Eingang in die AWMF-Leitlinien gefunden [2].

Die Produktinformationen der generell zur initialen Antikoagulation empfohlenen NMH und UFH sowie von Fondaparinux berücksichtigen den Aspekt der Niereninsuffizienz, indem bei Erreichen

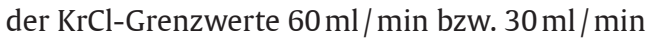
entweder

- generelle Vorsicht bei der Anwendung,

- zusätzliche Diagnostik (insbesondere Anti-XaMessungen),

- eine Dosisadaption (primär bei EnoxaparinGabe bei $\mathrm{KrCl}<30 \mathrm{ml} / \mathrm{min}$ ) oder

- bei einigen Wirkstoffen im Falle einer $\mathrm{KrCl}<30 \mathrm{ml} / \mathrm{min}$ der generelle Verzicht auf den Einsatz

empfohlen werden ( $\triangleright$ Abb. 2).
Insgesamt 12 der in die Übersicht eingeschlossenen Publikationen diskutieren Niereninsuffizienz als unabhängigen Risikofaktor für das Auftreten von rezidivierenden VTE, Blutungen oder Mortalität, insbesondere bei der initialen Therapie mit $\mathrm{NMH}$. Tsai et al. zeigten in einer retrospektiven Analyse von 153518 Krankenhauspatienten, dass eine terminale Niereninsuffizienz ein unabhängiger Risikofaktor für Mortalität bei VTE-Patienten ist (Odds Ratio 1,4; 95\%-Konfidenzintervall $[1,3-1,5])[15]$.

In einer retrospektiven Analyse von 1509 Patientenakten kamen Parikh et al. zum Ergebnis, dass VTE-Patienten mit einer $\mathrm{KrCl}<30 \mathrm{ml} /$ min ein

- erhöhtes Rezidivrisiko (Hazard Ratio: 1,83, [1,03-3,25]),

- ein erhöhtes Risiko schwerer Blutungen (HR 2,30, [1,28-4,16]) sowie

- eine allgemein höhere Mortalität (HR: 1,70, [1,12-2,57])

während einer 3-jährigen Beobachtungszeit aufwiesen [12].

Falgá et al. verdeutlichten im Rahmen einer retrospektiven Analyse der bereits zitierten RIETERegisterdaten, dass eine $\mathrm{KrCl}<30 \mathrm{ml} / \mathrm{min} \mathrm{zu}$ einem höheren Risiko

- tödlicher LE (OR 6,5, [4,7-8,9]),

- tödlicher Blutungen (OR 4,6, [2,8-7,2]) und - der allgemeinen Mortalität (OR 4,2, [3,6-4,9]) bei VTE-Patienten führt [7].

Monreal et al. bestätigten das Ergebnis häufigerer fataler Lungenembolien (OR 7,2, [4,9-11,0]) und tödlicher Blutungen (OR 6,5, [2,7-16,0]) im Vergleich von VTE-Patienten mit einer geschätzten
Abb. 2 Produktinformationen für $\mathrm{NMH}$, UFH und Fondaparinux bei initialer Therapie von niereninsuffizienten TVT/ LE-Patienten 
glomerulären Filtrationsrate eGRF $<30 \mathrm{ml} / \mathrm{min} z u$ Patienten mit einer eGFR $>60 \mathrm{ml} / \mathrm{min}$ auf Basis einer weiteren RIETE-Analyse [11].

$\mathrm{Zu}$ vergleichbaren Schlussfolgerungen hinsichtlich des erhöhten Risikos von Blutungsereignissen bei einer eGFR $<30 \mathrm{ml} / \mathrm{min}$ kamen

- Spencer et al. in einer prospektiven Kohortenstudie (HR 1,85, [1,15-3,00]) [13],

- Ellis et al. in einer prospektiven Kohortenstudie (OR 5,685, [1,909-16,292]) [6] sowie

- Bauersachs et al. [4] und Clark et al. [5] in einschlägigen Übersichtsarbeiten.

Kooiman et al. kamen in einer weiteren niederländischen retrospektiven Analyse zum Ergebnis, dass das Risiko schwerer Blutungen bei Patienten mit einer $\mathrm{KrCl}<30 \mathrm{ml} / \mathrm{min}$ (Vergleich mit $\mathrm{KrCl}>60 \mathrm{ml} / \mathrm{min}$ ) insbesondere bei der initialen NMH-Therapie mit einer HR von 4,6 ([2,0-6,3]) im Vergleich zur prolongierten VKA-Therapie (HR 1,0, [0,2-4,5]) deutlich erhöht ist [9].

Lim et al. kamen zu dem Ergebnis, dass die Rate von Blutungsereignissen bei Patienten mit einer $\mathrm{KrCl} \leq 30 \mathrm{ml} / \mathrm{min}$ ca. doppelt so hoch ist wie die der Patienten mit einer $\mathrm{KrCl}>30 \mathrm{ml} / \mathrm{min}$ (5,0\% vs. $2,4 \%$, OR 2,25, [1,19-4,27], $\mathrm{p}=0,013$ ) [10]. Einen Anstieg der Blutungsraten mit steigendem Grad der Nierenfunktionseinschränkung sahen sowohl Thorevska et al. in einer retrospektiven Studie (Vergleich GFR $<60 \mathrm{ml} / \mathrm{min}$ vs. GFR > $60 \mathrm{ml} / \mathrm{min}$; HR 1,3, [1,0-1,6]) [14] als auch Hoffmann/Keller in einer Übersicht [8] wobei letztgenannte den Anstieg als exponentiell mit jeder schlechteren CKD-Klasse beschreiben.

14 der in diese Übersicht eingeschlossenen Publikationen thematisieren das antikoagulationsbezogene Blutungsrisiko der betrachteten Patientengruppe. Trujillo-Santos et al. berichten auf Basis der Daten des RIETE-Registers, dass VTEPatienten eine höhere Sterblichkeit innerhalb von 15 Tagen bei Behandlung mit UFH als bei NMH-Therapie aufwiesen. Für die drei Nierenfunktionsklassen waren die Ergebnisse im Einzelnen:

- $\mathrm{KrCl}>60 \mathrm{ml} / \mathrm{min}$ mit UFH versus NMH: 4,5\% vs. 2,4\% (OR 1,9, 95\%-Konfidenzintervall [1,3-2,8])

- $\mathrm{KrCl} 30-60 \mathrm{ml} / \mathrm{min}: 5,4 \%$ vs. 5,8\% (nicht signifikant unterschiedlich)

- $\mathrm{KrCl}<30 \mathrm{ml} / \mathrm{min}: 15,0 \%$ vs. $8,1 \%$ (OR 2,0, [1,13,9]).

Zusätzlich zeigten mit UFH behandelte niereninsuffiziente Patienten eine erhöhte Rate von tödlichen Lungenembolien

- $\mathrm{KrCl}>60 \mathrm{ml} / \mathrm{min}$ mit UFH versus NMH: $2,8 \%$ vs. $1,2 \%$ (OR 2,4, [1,4-4,2])

- $\mathrm{KrCl} 30-60 \mathrm{ml} / \mathrm{min}: 3,2 \%$ vs. $2,5 \%$ (nicht signifikant unterschiedlich)

- $\mathrm{KrCl}<30 \mathrm{ml} / \mathrm{min}: 5,7 \%$ vs. $2,4 \%$ (nicht signifikant unterschiedlich).
Die Rate schwerer Blutungen war ähnlich

- $\mathrm{KrCl}>60 \mathrm{ml} / \mathrm{min}$ mit UFH versus NMH: 1,7\% vs. $1,1 \%$ (nicht signifikant unterschiedlich)

- $\mathrm{KrCl} 30-60 \mathrm{ml} / \mathrm{min}: 2,9 \%$ vs. 3,2\% (nicht signifikant unterschiedlich)

- $\mathrm{KrCl}<30 \mathrm{ml} / \mathrm{min}: 2,4 \%$ vs. 3,3\% (nicht signifikant unterschiedlich) [30].

In einer retrospektiven Untersuchung unter 620 Patienten mit einer $\mathrm{KrCl}<60 \mathrm{ml} / \mathrm{min}$ zeigten Thorevska et al. Raten schwerwiegender Blutungsereignisse von 26,3 per 1000 Personenjahre für UFH und 20,7 per 1000 Personenjahre für Enoxaparin auf ( Incidence Rate Ratio: 0,79, [0,47-1,32]). Die Rate leichter Blutungsereignisse war unter Enoxaparin-Gabe bei Patienten mit einer GFR $<30 \mathrm{ml} / \mathrm{min}$ allerdings um $154 \%$ erhöht (IRR: 2,54, [1,01-6,36]) [14].

Zu einem erhöhten Risiko einer Enoxaparin-Therapie im Vergleich zu anderen Antikoagulanzien (RR 1,67, [1,12-2,50], p=0,01) kommen Hoffmann/Keller in einer Meta-Analyse von Patienten mit moderater Nierenfunktionseinschränkung [8]. Eine von Leizorovicz et al. ausgewertete klinischeStudie von TVT-Patienten, beider dieUFH- mit der NMH-Gabe (Tinzaparin) verglichen wurde, musste aufgrund einer erhöhten Mortalität im UFH-Arm abgebrochen werden. Dabei waren

- Blutungsraten (11,9\% vs. 11,9\%, RR 0,99, [0,631,57]) und

- Rezidive (3,4\% vs. 5,9\%, RR 1,77, [0,79-3,92], $\mathrm{p}=0,16)$

vergleichbar häufig in den beiden Behandlungsarmen. In einer post-hoc-Analyse zeigte sich, dass die unterschiedlichen Todesraten auf sonstige Komorbiditäten, die ungleich zwischen den Gruppen verteilt waren und nicht auf die Therapie $(p=0,28)$ der Patienten zurückgingen [31].

In einer prospektiven observationalen Studie kamen Lai et al. zum Ergebnis, dass die Gabe einer nicht-adjustierten therapeutischen EnoxaparinDosis bei niereninsuffizienten Patienten zu höheren Blutungsraten führt [32]. Dies wurde in den Übersichten von von Samama et al. [33] und Nutesco et al. [26] bestätigt. Lim et al. zeigen in einer Meta-Analyse, dass die Blutungsraten bei Patienten mit einer GFR $<30 \mathrm{ml} / \mathrm{min}$ vs. Patienten mit einer GFR $>30 \mathrm{ml} /$ min unter standardisierter Enoxaparin-Dosis deutlich erhöht waren (8,3\% vs. $2,4 \%$, OR 3,88, [1,78-8,45]) im Vergleich zu einer empirisch adaptierten Dosis Enoxaparin (0,9\% vs. $1,9 \%$, OR 0,58, [0,09-3,78]) [10].

In einer weiteren Übersicht kamen Crowther et al. zum Ergebnis, dass Unterschiede in der Akkumulation von NMH bestehen [34]. Dabei neige das NMH Tinzaparin aufgrund des im Vergleich höheren durchschnittlichen Molekulargewichts weniger zur Akkumulation. Damit in Übereinstimmung sahen Nagge et al. in ihrer Übersicht keine Evidenz, die die $\mathrm{KrCl}-$ Grenze von $30 \mathrm{ml} / \mathrm{min}$ rechtfertigt. 
Ihrer Meinung nach beständen keine Hinweise auf Akkumulation von Tinzaparin bis zu einer $\mathrm{KrCl} \geq 20 \mathrm{ml} / \mathrm{min}$ [35].

Bauersachs et al. verglichen Certoparin mit UFH bei der Thromboseprophylaxe bei Patienten mit einer $\mathrm{KrCl}<30 \mathrm{ml} / \mathrm{min}$. Sie schlussfolgern, dass die Effektivität beider Wirkstoffe vergleichbar ist (4,55\% vs. $4,21 \%$, OR 1,08, [0,5-2,37]), die Certoparin-Gabe aber zu einer geringeren Blutungshäufigkeit führt (9,52 vs. $3,54 \%$, OR 2,87, [1,70-4,83]) [36].

Ageno et al. zeigten in einer prospektiven Beobachtungsstudie von 206 chronisch kranken Patienten mit einer $\mathrm{KrCl} 20-50 \mathrm{ml} / \mathrm{min}$, die 6-15 Tage mit niedrigdosiertem Fondaparinux zur Thromboseprophylaxe behandelt wurden, dass lediglich ein schwerer Blutungsfall auftrat. Sie schließen daraus die Sicherheit der niedrigdosierten Fondaparinux-Therapie [37].

Bauersachs et al. argumentieren in einer weiteren pharmakologischen Publikation, dass auch bei neuen oralen Antikoagulanzien aufgrund der pharmakologischen Eigenschaften dieser Wirkstoffe eine Akkumulation bei Niereninsuffizienz zu erwarten ist [38]. In der EINSTEIN-Studie zur Wirksamkeit und Sicherheit von Rivaroxaban konnte gezeigt werden, dass es unabhängig von der NF keine signifikanten Unterschiede bei diesen Endpunkten zwischen Rivaroxaban und Enoxaparin/Vitamin-K-Antagonisten-Therapie gibt. So war die Wirksamkeit (rezidive venöse Thromboembolie) in allen drei Nierenfunktionsklassen vergleichbar:

- GFR > $80 \mathrm{ml} / \mathrm{min}: 1,6 \%$ vs. $2,6 \%$

- GFR $50-80 \mathrm{ml} / \mathrm{min}: 3,1 \%$ vs. $3,5 \%$

- GFR $<50 \mathrm{ml} / \mathrm{min}: 4,2 \%$ vs. $5,0 \%$, alle nicht signifikant.

Ähnliches galt auch für das Auftreten von Blutungsereignissen

- GFR > $80 \mathrm{ml} / \mathrm{min}: 7,5 \%$ vs. $7,4 \%$

- GFR $50-80 \mathrm{ml} / \mathrm{min}: 9,2 \%$ vs. $10,3 \%$

- GFR $<50 \mathrm{ml} / \mathrm{min}: 10,8 \%$ vs. 7,8\%, alle nicht signifikant [17].

Lediglich 7 der eingeschlossenen Publikationen widmeten sich dem Thema der Behandlung von niereninsuffizienten VTE-Patienten unter Praxisbedingungen. In einer italienischen Studie berichteten Dentali et al., dass in Bezug auf die Entlassmedikation nach einem stationären Aufenthalt eine Thromboseprophylaxe bei 61,4\% der VTERisikopatienten verschrieben wurde. Dabei gab es keinen Unterschied in der Verschreibungsrate oder der Dosis für Patienten mit oder ohne Niereninsuffizienz [39].

Eine Patientenaktenanalyse von Salomon et al., die nicht auf VTE-Patienten beschränkt war, zeigte, dass ca. $20 \%$ aller Patienten mit Niereninsuffizienz (definiert als Serum-Kreatinin $\geq 1,7 \mathrm{mg} / \mathrm{dl}$ ) keine gewichtsadaptierte Dosierung, wie in den
Fachinformationen der jeweiligen Präparate empfohlen, erhielten. Ein ähnlicher Prozentsatz an Patienten erhielt Wirkstoffe, die nicht für die Anwendung bei niereninsuffizienten Patienten zugelassen waren [40].

Cook et al. haben in einer retrospektiven Studie unter 524 VTE Patienten in 36\% der Fälle eine zu geringe Dosis niedermolekularer Heparine beobachtet, wobei $28 \%$ allein auf adipöse Patienten entfielen [41].

Aus den Ergebnissen eines internationalen Surveys, an dem 257 Krankenhäuser teilnahmen, schlussfolgern Barras et al., dass einer der wichtigsten Gründe für die falsche Dosierung von Arzneimitteln bei niereninsuffizienten Patienten krankenhausindividuelle Dosierregeln/Dosierprotokolle seien, die sich von den Fachinformationsempfehlungen unterschieden [42].

Sevestre et al. analysierten die Antikoagulationstherapie von Krebspatienten mit einem TVT/LEEreignis in Frankreich. Sie wiesen nach, dass ein Großteil der Patienten (98\%) eine initiale Antikoagulationsbehandlung erhält, dieser Prozentsatz allerdings auf $62 \%$ bei der ebenfalls empfohlenen langfristigen Therapie fällt. Bei niereninsuffizienten Patienten erhalten lediglich $25 \%$ eine adäquate Therapie [43].

Tiryaki et al. schlussfolgern in einer retrospektiven Studie, dass Niereninsuffizienz zu einer niedrigeren Rate verschriebener Antikoagulationstherapie bei Patienten mit einem VTE-Ereignis oder akutem Koronarsyndrom führt [30].

In der bereits erwähnten Patientenaktenstudie von Boettger et al. wurde für Deutschland gezeigt, dass ca. $47,1 \%$ der stationär behandelten VTE-Patienten mit einer $\mathrm{KrCl}<30 \mathrm{ml} /$ min keine leitlinien- bzw. produktinformationsgerechte initiale Antikoagulationstherapie erhalten. Bei 5,6\% der Patienten wurden Arzneimittel angewendet, die nicht für diese Patientengruppe zugelassen sind. In weiteren 6,2\% der Fälle erhielten die VTE-Patienten keine Antikoagulation. Bei 1,8\% der Patienten mit einer UFH-Therapie wurden aPTT-Werte nicht gemessen bzw. zumindest nicht dokumentiert. In 6,5\% der Fälle, in denen die initiale Antikoagulationstherapie mit Enoxaparin erfolgte, war in der Patientenakte keine Information zum Patientengewicht dokumentiert, was eine gewichtsadaptierte Enoxaparin-Dosierung praktisch ausschließt. Bei weiteren $27,0 \%$ der Enoxaparin-behandelten VTEPatienten mit einer $\mathrm{KrCl}<30 \mathrm{ml} /$ min erfolgte eine zu hohe Dosierung des Wirkstoffs [29].

\section{Diskussion}

Ziele und Ergebnisse der Übersicht | Die in dieser Übersicht dargestellten Daten zeigen, dass die Prä- 
valenz einer moderaten Niereninsuffizienz $(\mathrm{KrCl}<60 \mathrm{ml} / \mathrm{min})$ bzw. starken Niereninsuffizienz $(\mathrm{KrCl}<30 \mathrm{ml} / \mathrm{min})$ bei VTE-Patienten als hoch einzuschätzen ist. Die deutlichen Unterschiede in den berichteten Prävalenzraten sind u.a. auf die unterschiedliche Schätzung der NF zurückzuführen. Die zur Verfügung stehenden Schätzformeln kommen zum Teil zu deutlich differierenden Ergebnissen [44-47]. Ganz wesentlich sind die verschiedenen Prävalenzen jedoch auf die Unterschiede in den Charakteristika der untersuchten Patientenkollektive zurückzuführen (Alter, Geschlecht, Gesundheitsstatus etc.).

Die publizierten Daten zeigen nahezu einheitlich, dass eine stark eingeschränkte NF, definiert als $\mathrm{KrCl}<30 \mathrm{ml} / \mathrm{min}$, ein unabhängiger Prädiktor für Mortalität und fatale LE, evtl. auch für schwere Blutungen, bei der Therapie von VTE-Patienten ist. Dabei ist NF vermutlich auch schon ein Prädiktor für das Auftreten einer VTE. Ein exakt beschriebener pathophysiologischer Zusammenhang zwischen VTE und eingeschränkter NF existiert außer beim nephrotischen Syndrom (Verlust gerinnungshemmender Plasmafaktoren) bislang nicht. Erhöhte Blutungsraten unter Therapie lassen sich am ehesten durch Retention von Antikoagulanzien bei verringerter Ausscheidung erklären $[22,48]$.

Die Daten zeigen auch, dass zahlreiche Antikoagulanzien zur Akkumulation neigen und so beispielhaft gut darstellbar ist, dass nicht dosisangepasstes Enoxaparin in der VTE-Therapie höhere Blutungsraten zur Folge hat. $\mathrm{Zu}$ anderen $\mathrm{NMH}$ und auch zu UFH gibt es weitaus weniger aussagekräftige Daten. Die fehlende Verfügbarkeit dieser Daten sollte allerdings nicht falsch als Nachweis einer höheren Effektivität / Sicherheit dieser Wirkstoffe im Vergleich zu Enoxaparin interpretiert werden. Allein zur Sicherheit von Certoparin und Tinzaparin konnten eigene Studien in der betrachteten speziellen Patientengruppe identifiziert werden.

Keine der identifizierten Studien zeigt Effektivitäts- und/oder Sicherheitsvorteile der UFH- im Vergleich zur NMH-Therapie bei VTE-Patienten mit stark eingeschränkter NF. Vielmehr kann nach aktueller Studienlage deren Nachteiligkeit angenommen werden. Die Evidenz beruht allerdings auf observationalen Studien bzw. Registerdaten. In einer randomisierten klinischen Studie konnte diese Schlussfolgerung bislang nicht abgeleitet werden. Dennoch stellt sich auf Basis dieser Ergebnisse die Frage, inwiefern die Leitlinienempfehlung einer UFH-Therapie bei VTE-Patienten mit einer $\mathrm{KrCl}<30 \mathrm{ml} /$ min kritisch zu prüfen ist [2]. Damit übereinstimmend zeigen reale Versorgungsdaten, dass ca. 46,6-67,0\% der VTE-Patienten in deutschen Akutkrankenhäusern mit NMH initial behandelt werden $[14,29,41]$.
Limitationen | Bei der Interpretation der Ergebnisse dieser Übersicht sollten die methodischen Limitationen berücksichtigt werden. Zum einen adressiert die Übersicht mit der Betrachtung der initialen therapeutischen Antikoagulation lediglich einen Aspekt der Versorgung von niereninsuffizienten VTE-Patienten. Andere Elemente der Therapie der Patienten wurden nicht betrachtet. In Bezug auf die Identifikation von niereninsuffizienten Patienten in den eingeschlossenen Studien ist zudem festzustellen, dass eine große methodische Vielfalt zu beobachten war. Dies betraf

- den Zeitpunkt und die Häufigkeit der Nierenfunktionsmessung,

- die verwendete Schätzformel und

- die definierte $\mathrm{KrCl}-\mathrm{Grenze}$.

Hinzu kommt, dass ein Großteil der in dieser Übersicht dargestellten Evidenz aus observationalen prospektiven Studien, Registerdaten oder sonstigen retrospektiven Datenanalysen stammte. Der Vergleich von Effektivitäts- und Sicherheitsdaten verschiedener zur Auswahl stehender Wirkstoffe (insbesondere UFH vs. NMH vs. Fondaparinux vs. neue orale Antikoagulanzien) bei niereninsuffizienten VTE-Patienten in randomisierten klinischen Studien steht bislang noch aus.

\section{Konsequenz für Klinik und Praxis}

- Es ist davon auszugehen, dass mindestens 5-7\% der initial behandelten VTE-Patienten eine schwer eingeschränkte $\mathrm{NF}(\mathrm{KrCl}<30 \mathrm{ml} / \mathrm{min})$ aufweisen.

- Diese Patienten sind mit besonderer Vorsicht hinsichtlich Auswahl und Dosierung der Antikoagulanzien zu behandeln. Die breite Evidenz für die höhere LE-bezogene Mortalität und Rezidivrate dieser Patientengruppe zeigt aber auch, dass eine initiale Antikoagulationstherapie bei diesen Patienten in jedem Fall unmittelbar nach Diagnosestellung einzuleiten ist.

- Die aktuellen AWMF-Leitlinien geben für dieses Patientenkollektiv einen ersten Überblick. Allerdings ist neben der revidierbedürftigen Empfehlung von UFH als Option erster Wahl eine Kenntnis der Fachinformation der Substanzen unerlässlich, da diese nähere Auskünfte zu Dosierungsschemata bzw. zur Kontraindikation einiger Substanzen in diesem Kollektiv enthalten.

\section{Literatur}

1 Blix HS, Viktil KK, Moger TA Reikvam A. Use of renal risk drugs in hospitalized patients with impaired renal function - an underestimated problem? Nephrol Dial Transplant 2006; 21: 3164-3171

2 Deutsche Gesellschaft für Angiologie. Diagnostik und Therapie der Venenthrombose und der Lungenembolie. AWMF-Leitlinien-Register Nr. 065/002. 
http://www.awmf.org/uploads/tx szleitlinien/065-002_S2_Diagnostik_Therapie Venenthrombose_Lungenembolie_2010abgelaufen.pdf

Letzter Zugriff am 27.07.2015

3 Moysidis T, Santosa F, Stallinger C, Kröger K. Cranial and non-cranial embolism: incidence in hospitalised patients in Germany. J Thromb Thrombolysis 2013; 36: 369-374

4 Bauersachs RM. Use of anticoagulants in elderly patients. Thromb Res 2012; 129: 107-115

5 Clark NP. Low-molecular-weight heparin use in the obese, elderly, and in renal insufficiency. Thromb Res 2008; 123 (Suppl. 1): S58-S61

6 Ellis $\mathrm{MH}$, Hadari R, Tchuvrero $\mathrm{N}$ et al. Hemorrhagic complications in patients treated with anticoagulant doses of a low molecular weight heparin (enoxaparin) in routine hospital practice. Clin Appl Thromb Hemost 2006; 12: 199-204

7 Falgá C, Capdevila JA, Soler S et al. Clinical outcome of patients with venous thromboembolism and renal insufficiency. Findings from the RIETE registry. Thromb Haemost 2007; 98 . 771-776

8 Hoffmann P, Keller F. Increased major bleeding risk in patients with kidney dysfunction receiving Enoxaparin: a meta-analysis. Eur J Clin Pharmacol 2012; 68: 757-765

9 Kooiman J, den Exter PL, Cannegieter SC et al. Impact of chronic kidney disease on the risk of clinical outcomes in patients with cancer-associated venous thromboembolism during anticoagulant treatment. J Thromb Haemost 2013; 11: 1968-1976

10 Lim W, Dentali F, Eikelboom JW, Crowther MA. Meta-analysis: low-molecular-weight heparin and bleeding in patients with severe renal insufficiency. Ann Intern Med 2006; 144: 673-684

11 Monreal M, Falgá C, Valle R et al. Venous thromboembolism in patients with renal insufficiency: findings from the RIETE Registry. Am J Med 2006; 119: 1073-1079

12 Parikh AM, Spencer FA, Lessard D et al. Venous thromboembolism in patients with reduced estimated GFR: a population-based perspective. Am J Kidney Dis 2011; 58: 746-755

13 Spencer FA, Gore JM, Reed G et al. Venous thromboembolism and bleeding in a community setting. The Worcester Venous Thromboembolism Study. Thromb Haemost 2009; 101: 878-885

14 Thorevska N, Amoateng-Adjepong Y, Sabahi R et al. Anticoagulation in hospitalized patients with renal insufficiency: a comparison of bleeding rates with unfractionated heparin vs Enoxaparin. Chest 2004; 125: 856-863

15 Tsai ], Abe K, Boulet SL et al. Predictive accuracy of 29-comorbidity index for in-hospital deaths in US adult hospitalizations with a diagnosis of venous thromboembolism. PLoS One. 2013; 8: e70061

16 Botticelli Investigators. Efficacy and safety of the oral direct factor Xa inhibitor apixaban for symptomatic deep vein thrombosis. The Botticelli DVT dose-ranging study. J Thromb Haemost 2008; 6: 1313-1318

17 Bauersachs R, Berkowitz SD, Brenner B et al. Oral rivaroxaban for symptomatic venous thromboembolism. N Engl J Med 2010; 363: 2499-2510

18 Moher D, Liberati A, Tetzlaff ] et al. Preferred Reporting Items for Systematic Reviews and Meta-Analyses: The PRISMA Statement. Ann Intern Med 2009; 151: 264-269

19 Mahmoodi BK, Gansevoort RT, Næss IA, et al. Association of mild to moderate chronic kidney disease with venous thromboembolism: pooled analysis of five prospective general population cohorts. Circulation 2012; 126: 1964-1971
20 Folsom AR, Lutsey PL, Astor BC et al. Chronic kidney disease and venous thromboembolism: a prospective study. Nephrol Dial Transplant. 2010; 25: 3296-3301

21 Kumar G, Sakhuja A, Taneja A et al. Pulmonary embolism in patients with CKD and ESRD. Clin J Am Soc Nephrol 2012; 7: 1584-1590

22 Wattanakit K, Cushman M. Chronic kidney disease and venous thromboembolism: epidemiology and mechanisms. Curr Opin Pulm Med 2009; 15: 408-412

23 Cook D, Crowther M, Meade M et al. Deep venous thrombosis in medical-surgical critically ill patients: prevalence, incidence, and risk factors. Crit Care Med 2005; 33: 1565-1571

24 Königsbrügge O, Lötsch F, Zielinski C et al. Chronic kidney disease in patients with cancer and its association with occurrence of venous thromboembolism and mortality. Thromb Res 2014; 134: 44-49

25 Kurtal H, Schwenger V, Azzaro M et al. Clinical Value of Automatic Reporting of Estimated Glomerular Filtration Rate in Geriatrics. Gerontology 2009; 55: 288-295

26 Nutescu EA, Spinler SA, Wittkowsky A, Dager WE. Low-molecular-weight heparins in renal impairment and obesity: available evidence and clinical practice recommendations across medical and surgical settings. Ann Pharmacother 2009; 43: 1064-1083

27 Trujillo-Santos J, Schellong S, Falga C et al. Low-molecular-weight or unfractionated heparin in venous thromboembolism: the influence of renal function. Am J Med. 2013; 126: 425-434

28 Al-Dorzi HM, Al-Heijan A, Tamim HM et al. Renal failure as a risk factor for venous thromboembolism in critically Ill patients: a cohort study. Thromb Res 2013; 132: 671-675

29 Boettger B, Wehling M, Bauersachs RM et al. Initial anticoagulation therapy in patients with venous thromboembolism and impaired renal function: results of an observational study. J Public Health 2014; 22: 89-99

30 Tiryaki F, Nutescu EA, Hennenfent JA et al. Anticoagulation therapy for hospitalized patients: patterns of use, compliance with national guidelines, and performance on quality measures. Am J Health Syst Pharm 2011; 68: 1239-1244

31 Leizorovicz A, Siguret V, Mottier D et al. Safety profile of tinzaparin versus subcutaneous unfractionated heparin in elderly patients with impaired renal function treated for acute deep vein thrombosis: the Innohep ${ }^{\circledR}$ in Renal Insufficiency Study (IRIS). Thromb Res 2011; 128: 27-34

32 Lai S, Barbano B, Cianci R et al. The risk of bleeding associated with low molecular weight heparin in patients with renal failure. G Ital Nefrol. 2010; 27: 649-654

33 Samama MM. Use of low-molecular-weight heparins and new anticoagulants in elderly patients with renal impairment. Drugs Aging 2011; 28: 177-193

34 Crowther M, Lim W. Low molecular weight heparin and bleeding in patients with chronic renal failure. Curr Opin Pulm Med 2007; 13: 409-413

35 Nagge J, Crowther M, Hirsh J. Is impaired renal function a contraindication to the use of lowmolecular-weight heparin? Arch Intern Med 2002; 162: 2605-2609

36 Bauersachs RM. New oral anticoagulants and chronic kidney disease. Internist (Berl). 2012; 53: 1431-1444

37 Ageno W, Riva N, Noris P et al. Safety and efficacy of low-dose fondaparinux $(1.5 \mathrm{mg})$ for the prevention of venous thromboembolism in acutely ill medical patients with renal impairment: the 
FONDAIR study. J Thromb Haemost 2012; 10 : 2291-2297

38 Bauersachs R, Schellong SM, Haas S et al. CERTIFY: prophylaxis of venous thromboembolism in patients with severe renal insufficiency. Thromb Haemost 2011; 105: 981-988

39 Dentali F, Riva N, Gianni M et al. Prevalence of renal failure and use of antithrombotic prophylaxis among medical inpatients at increased risk of venous thromboembolic events. Thromb Res 2008; 123: 67-71

40 Salomon L, Deray G, Jaudon MC et al. Medication misuse in hospitalized patients with renal impairment. Int I Qual Health Care 2003; 15 : 331-335

41 Cook LM, Kahn SR, Goodwin J, Kovacs MJ. Frequency of renal impairment, advanced age, obesity and cancer in venous thromboembolism patients in clinical practice. J Thromb Haemost 2007; 5: 937-941

42 Barras MA, Kirkpatrick CM, Green B. Current dosing of low-molecular-weight heparins does not reflect licensed product labels: an international survey. Br J Clin Pharmacol 2010; 69: 520-528

43 Sevestre MA, Belizna C, Durant C et al. Compliance with recommendations of clinical practice in the management of venous thromboembolism in cancer: the CARMEN study. J Mal Vasc 2014; 39: 161-168

44 Chambers JT, Chambers SK, Schwartz PE. Correlation between measured creatinine clearance and calculated creatinine clearance in ovarian cancer patients. Gynecol Oncol 1990; 36: 66-68

45 Levey AS, Bosch JP, Lewis JB et al. A more accurate method to estimate glomerular filtration rate from serum creatinine: a new prediction equation.
Modification of Diet in Renal Disease Study Group. Ann Intern Med 1999; 130: 461-470

46 Lim WH, Lim EM, McDonald S. Lean body mass-adjusted Cockcroft and Gault formula improves the estimation of glomerular filtration rate in subjects with normal-range serum creatinine. Nephrology (Carlton) 2006; 11: 250-256

47 Michels WM, Grootendorst DC, Verduijn M et al. Performance of the Cockcroft-Gault, MDRD, and new CKD-EPI formulas in relation to GFR, age, and body size. Clin ] Am Soc Nephrol 2010; 5: 1003-1009

48 Cook DJ, Douketis ], Arnold D, Crowther MA. Bleeding and venous thromboembolism in the critically ill with emphasis on patients with renal insufficiency. Curr Opin Pulm Med 2009; 15: 455-462

\section{Interessenkonflikt}

Die Erstellung der Übersichtsarbeit wurde durch die Leo Pharma $\mathrm{GmbH}$ finanziell unterstützt.

Martin Wehling hat in den letzten drei Jahren

Beraterhonorare von Sanofi-Aventis, Novartis, Takeda, Roche, Pfizer, Bristol-Myers, Daichii-Sankyo, Lilly, Novo-Nordisk, Shire und LEO Pharma erhalten. Rupert Bauersachs und Thomas Wilke haben in den letzten drei Jahren Beraterhonorare von Boehringer Ingelheim Pharma, Bayer, Bristol Myers Squibb und Leo Pharma erhalten.

Steffen Amann hat in den letzten drei Jahren Beraterhonorare von Boehringer Ingelheim Pharma, B. Braun, Bristol Myers Squibb, MSD International, Novartis Pharma, Pfizer Pharma und Leo Pharma erhalten. 